\title{
Flexure Design Rules for Carbon Fiber Microrobotic Mechanisms
}

\author{
S. Avadhanula, R. S. Fearing \\ Department of Electrical Engineering and Computer Science \\ University of California, Berkeley \\ Email: \{srinath, ronf $\} @ e e c s . b e r k e l e y . e d u$
}

\begin{abstract}
Mechanisms utilizing rigid links and relatively small flexural joints are very suitable for fabrication at the meso scale. The Micromechanical Flying Insect (MFI) project at UC Berkeley has developed a simple fabrication method which works very well at the scale where the links are a few millimeters in length and the flexures are few hundred microns in length. Previous analysis has concentrated on the geometry of the composite material links for creating rigid links. Recently, we have found that for useful performance, detailed analysis is required of the flexures also. This paper presents a brief analysis of the issues involved in the design of the flexural components of such mechanisms.
\end{abstract}

\section{INTRODUCTION}

Flexural mechanisms have recently been used in increasingly many applications [1], [2] and have come under a lot of analysis [3], [4]. Some of the basic design issues involved in the fabrication of small length flexure mechanisms has been treated previously in [5]. Researchers have also studied various kinds of flexures for obtaining rotational motion from the point of view of optimal force transmission [6]. In this paper, we present an application of some of these general principles to a novel fabrication technology which was developed at UC Berkeley during the Micromechanical Flying Insect (MFI) project [7]. This technique is ideally suited to fabricating mechanisms at the millimeter scale. Fig. 1 shows some microrobots which have been constructed using this technology. The details of this new fabrication method have been elaborated in [8]. Briefly, the method consists of creating link/joint pairs by sandwiching polyester between layers of carbon fiber. By utilizing "folds" in the design, the planar sandwiched layer can be folded into a wide variety of 3D mechanisms [9]. Related work is concentrating on methods of automatically folding the mechanisms [10] and embedding wiring inside the flexures themselves [11]. The various performance aspects of the CF links have been elaborated in [8]. Various dynamic features of the MFI mechanism have been analyzed extensively previously [12], [13]. Over the course of the last few years using this new fabrication technique, we have come across many general design rules

This work was funded by NSF IIS-0083472, ONR MURI N00014-98-10671 and DARPA for the design of the flexural components of the mechanism. This paper presents these design rules, which we believe will be widely applicable in the construction of mechanisms using this procedure. For this paper, we use two flexural mechanisms currently used in the MFI project as driving examples. The first is a simple fourbar mechanism which is designed to amplify the motion of the PZT actuators by about $3000 \mathrm{rads} / \mathrm{m}$. The second is a 3D five-bar mechanism with $2 \mathrm{DOF}$ which is meant to couple the rotations of the two fourbars into a composite flapping and rotation (Fig. 2).

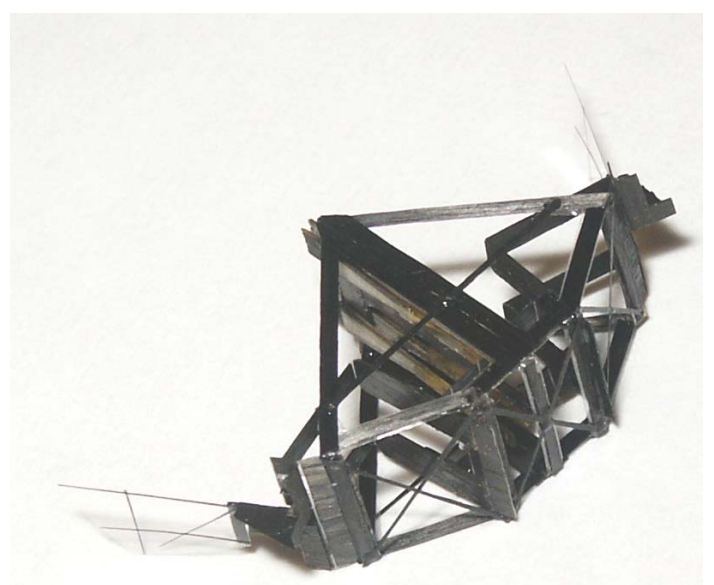

(a) Latest airframe structure of the Micromechanical Flying Insect

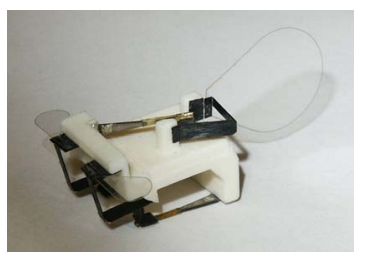

(b) Biomimetic Fishbot [14]

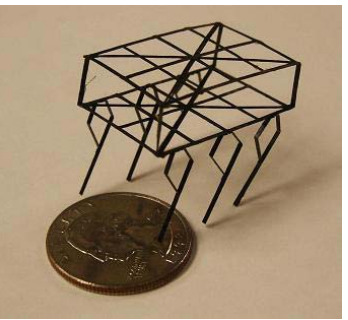

(c) Microrobotic Crawler
Fig. 1. Various mechanisms constructed using carbon fiber microfabrication techniques. 


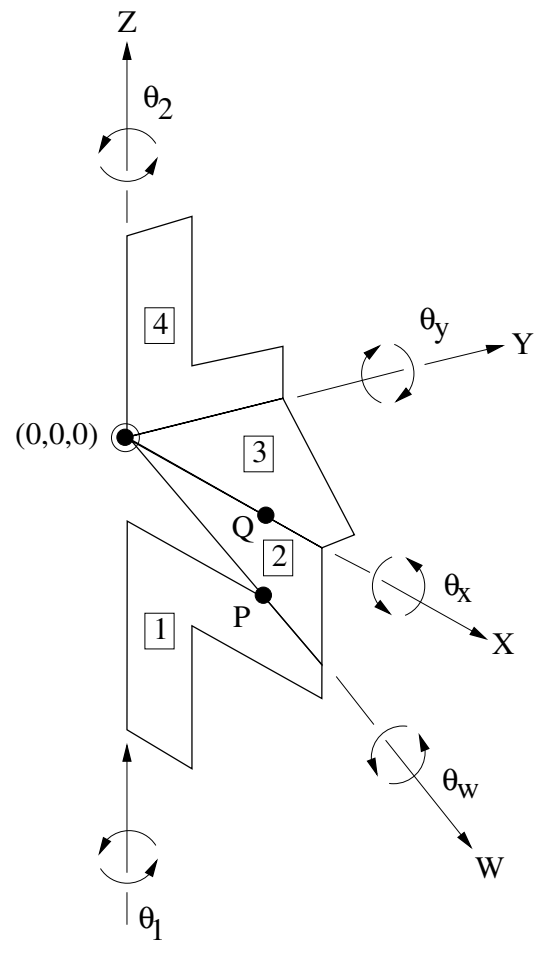

Fig. 2. Kinematic diagram of the MFI differential

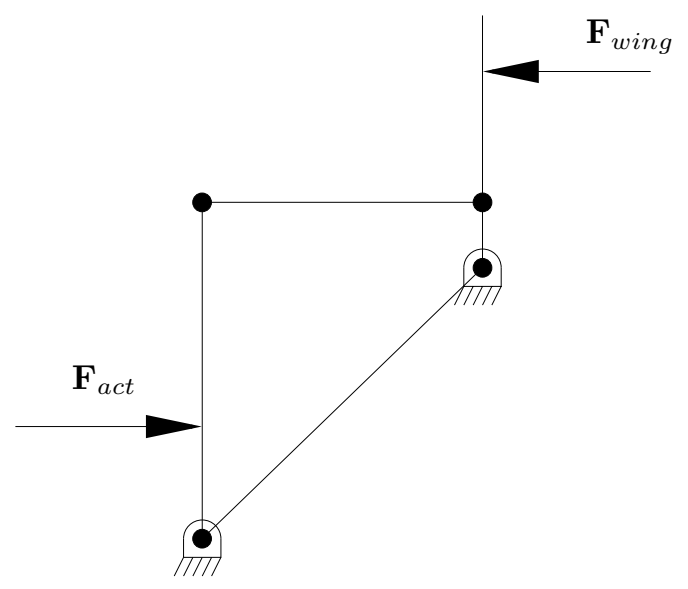

Fig. 3. Kinematic diagram of a simple fourbar mechanism showing the typical external forces acting on it.

The paper is organized as follows: Section II reviews a method for calculating the force transmitted through the flexures of a planar flexural mechanism which is subjected to a matched set of external forces. From this analysis, we derive a method for calculating the orientation of the flexures which leads to minimal serial stiffness. In Section III, we analyze the mechanism for the possibility of buckling in the flexures and design them for the required force transmission. Section IV analyses the parallel stiffness of the mechanism assuming no buckling in the flexures. We examine the effect which pre-stressing in the flexures has on the overall stiffness of the mechanism. We also qualitatively examine the effect of misalignment on the stiffness of a flexural mechanism.

In the above discussion, "parallel stiffness" refers to the force $F_{a c t}$ required to drive the input joint (Fig. 3) with all the links left free to rotate. For planar mechanisms like fourbars, this stiffness depends purely on the stiffness of the flexures. As we will later show, for 3D mechanisms, we also need to be very careful about alignment of the flexures. "Serial stiffness" refers to the free movement possible at the output link of the fourbar when the input link is fixed. In Fig. 3, this refers to the motion caused by $F_{\text {wing }}$ when the input link is fixed.

\section{FORCE ANALYSIS}

In this section we do a force analysis of the fourbar and give a systematic method for choosing the correct flexure orientations. To see why this is important, we focus first on a simple flexural setup. Consider two basic singular mechanisms with the flexures oriented differently (Fig. 4(a),(b)). If the flexures were ideal pin joints, then in both cases, the force $F$ would not cause any motion at all, i.e the two configurations would be infinitely stiff. If we perform a small motion FEA analysis of the two configurations, we find that the stiffness in the first case is almost an order of magnitude more than the second. This result implies that it is advantageous to have the flexures oriented in such a manner that the force transmitted through them always points along their length.

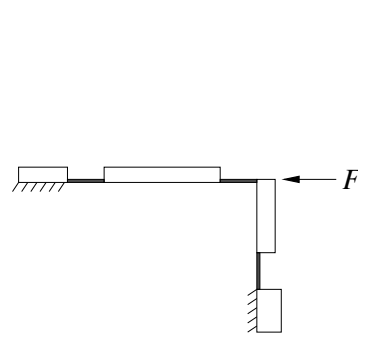

(a)

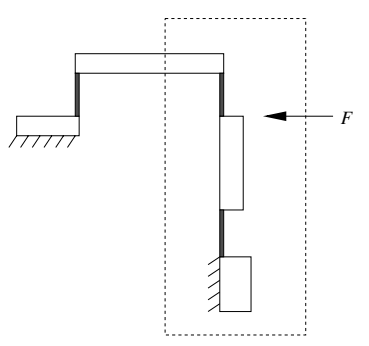

(b)
Fig. 4. FEA analysis of two simple flexural mechanisms.

To utilize this knowledge of flexural hinges, we first need to find out the direction of the forces transmitted through the various flexures for the typical external forces acting on the mechanism.

Fig. 3 shows the typical forces acting on the fourbar mechanism of the MFI. Here $\mathbf{F}_{\text {wing }}$ is the wing force acting on the output link of the fourbar and $\mathbf{F}_{a c t}$ is the actuator force acting on the input link. For this analysis, we make the following simplifying assumptions. First we assume that each flexure is an ideal pin joint with a torsional spring. Thus it is capable of transmitting both forces and moments. However, the transmitted forces are independent of the rotation of the flexure, while the transmitted moment follows directly from the rotation. Next, we assume that the links are moving quasistatically, i.e, the links have no acceleration. This implies that 


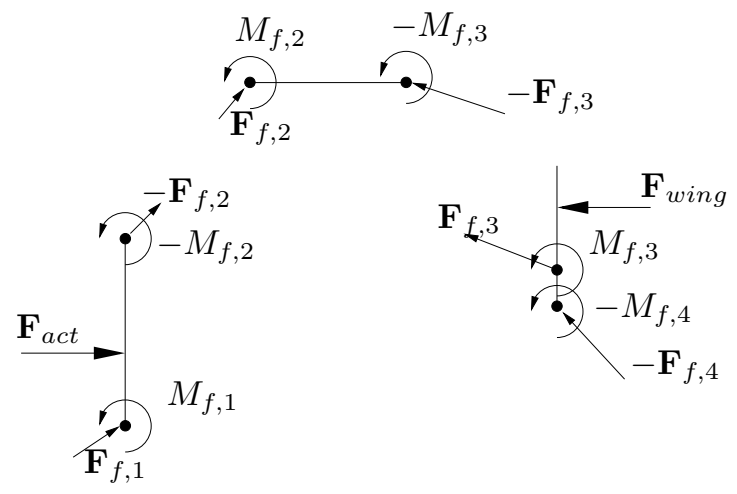

Fig. 5. Exploded view of the fourbar links showing the flexure forces acting on the links

given a fourbar configuration (specified by the link angles) and a force $\mathbf{F}_{\text {wing }}$ acting on the output link, we need to also specify a unique blocking force $\mathbf{F}_{a c t}$ to retain the fourbar at the given configuration. Since we know the way in which the actuator is attached to the fourbar, we will assume that the direction of $\mathbf{F}_{a c t}$ is known relative to the input link. The problem statement therefore becomes: Given a force $\mathbf{F}_{\text {wing }}$ acting on the output link of the fourbar, find the four vector forces transmitted through the fourbar flexures and also find the magnitude of the required blocking force $\mathbf{F}_{a c t}$. Thus we need to solve for 9 unknown quantities.

Fig. 5 shows an exploded view of the fourbar links showing the forces and moments acting on the various links. For our problem, we have to solve for the vectors $\mathbf{F}_{f, i}, i=1, \ldots 4$ and the scalar, $\left|\mathbf{F}_{a c t}\right| . \mathbf{F}_{f, i}$ here represents the force transmitted via the $i^{t h}$ flexure from the $(i-1)^{t h}$ to the $i^{t h}$ link. Similarly, $M_{f, i}$ represents the moment transmitted to the $i^{t h}$ link via the flexure connecting it to the $(i-1)^{t h}$ link. The forces and moments have to satisfy the following equilibrium equations for each of the link they act upon:

$$
\begin{gathered}
\mathbf{F}_{f, i}-\mathbf{F}_{f, i+1}+\mathbf{F}_{e x t}^{i}= \\
\left(\begin{array}{c}
\mathbf{F}_{f, i} \times r_{1}^{i}-\mathbf{F}_{f, i+1} \times r_{2}^{i} \\
+M_{f, i}-M_{f, i+1}+M_{e x t}^{i}
\end{array}\right)=0 \\
i=1, \ldots, 3
\end{gathered}
$$

Thus there are 9 scalar equilibrium equations involving 9 unknowns.

In the above equation $r_{1}^{i}$ and $r_{2}^{i}$ are the moment arms of $\mathbf{F}_{f, i}$ and $-\mathbf{F}_{f, i+1}$ about the CG of the $i^{t h}$ link. Note that we can simplify the solution method of (1) and (2) by proceeding with link 2 which has no external forces acting on it. This solution method for fourbars is well-known and will not be further elaborated upon here. See [3] for similar analysis. Here we present the final results of the force analysis. Fig. 6 shows the forces transmitted through the various flexures superimposed on the fourbar geometry for various configurations.
These forces were calculated in response to a constant force $\mathbf{F}_{\text {wing }}$ acting normal to the output link of the fourbar at all these configurations.

Fig. 7 shows how the transmitted flexural forces vary with the configuration of the fourbar. At the extremes, where the fourbar exhibits "stiffening" in the actuator coordinates, we see that we require a much larger $\mathbf{F}_{a c t}$ to balance the same wing force $\mathbf{F}_{\text {wing }}$.

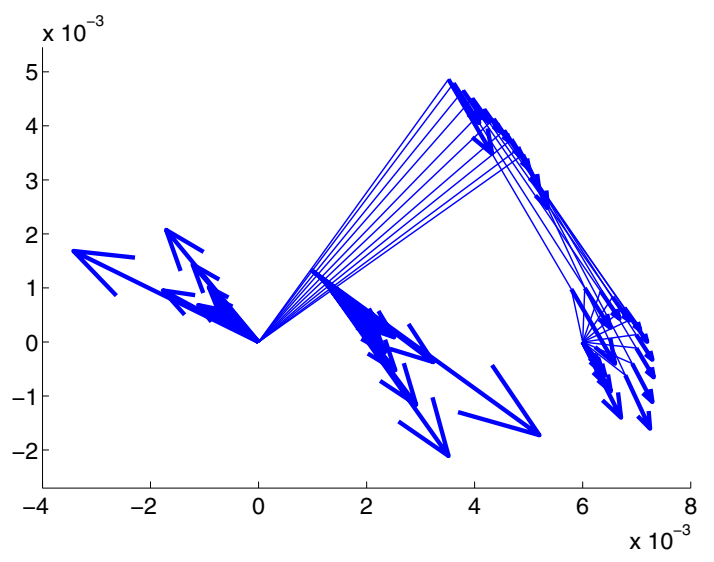

Fig. 6. Forces transmitted through the fourbar flexures

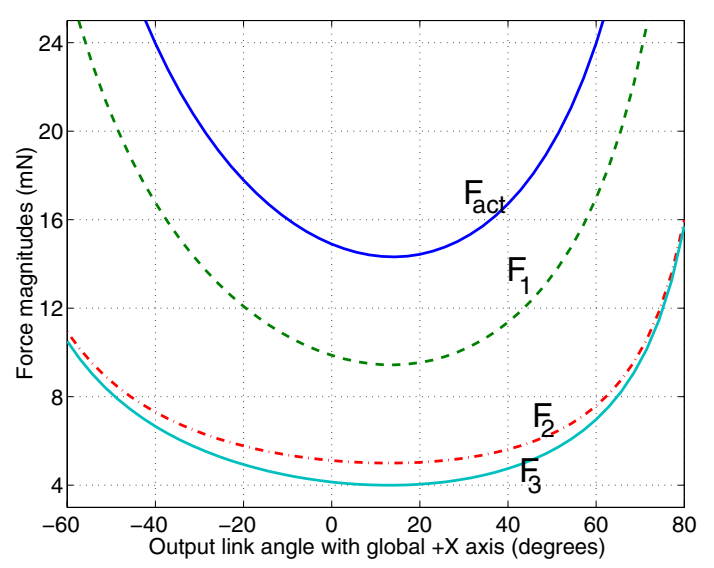

Fig. 7. Variation of transmitted Flexural Forces

This analysis tells us the direction in which the forces transmitted via the various flexures. This tells us the best way to orient the various flexures in the fourbar during fabrication for the most efficient force transmission. Combined with various fabrication limitations, this gives the fabricated configuration of our latest fourbar as shown in Fig. 8.

Note how all the flexures are oriented to be along the mean direction of the transmitted forces. We have experimentally measured the serial stiffness of the optimized fourbar mechanism and found it to be well over $4000 \mathrm{~N} / \mathrm{m}$. This is a huge 


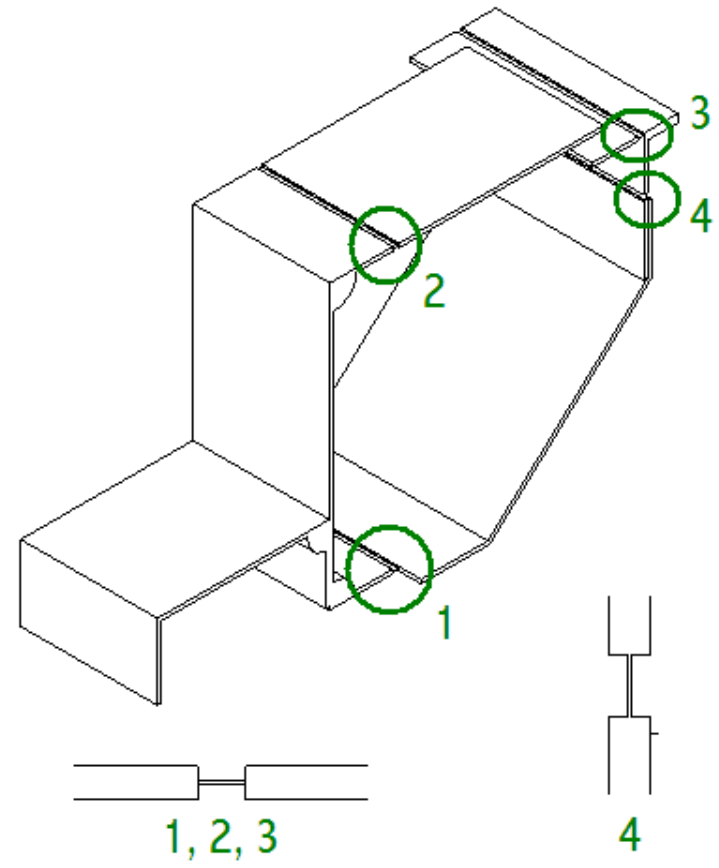

Fig. 8. Latest MFI Fourbar design

improvement from the serial stiffness of previous fourbars which had a measured serial stiffness of about $1000 \mathrm{~N} / \mathrm{m}$.

\section{BUCKLING STRENGTH OF THE FLEXURES}

The force analysis of the fourbar also allows us to calculate the maximum forces which can be transmitted through the fourbar. Alternatively, it allows us to design the fourbar flexures to match a given maximum force which needs to be blocked by the actuator. For the MFI, the maximum blocking force of the actuator is about $100 \mathrm{mN}$. This allows us to calculate the maximum forces being transmitted through the various flexures. Using the previous analysis, we find that $\left|\mathbf{F}_{1}\right|=65 \mathrm{mN},\left|\mathbf{F}_{2}\right|=\left|\mathbf{F}_{3}\right|=35 \mathrm{mN}$ and $\left|\mathbf{F}_{4}\right|=27 \mathrm{mN}$ for $\left|\mathbf{F}_{a c t}\right|=100 \mathrm{mN}$. This force configuration is able to withstand a aerodynamic force of $7 \mathrm{mN}$ applied at the centroid of the wing.

We want to design the flexures to not buckle under these kinds of loads. The maximum force which a flexure of length $l$ can withstand before buckling is given by

$$
F_{c r}=\frac{E I \pi^{2}}{L^{2}},
$$

where $E$ is the Young's modulus, $I$ is the moment of area and $L$ is the length. In practice, we will design with a factor of safety of 5 since the flexures are not always exactly parallel to the direction of the transmitted force. For our flexures, fabrication constraints restrict us to flexure lengths of $125 \mu \mathrm{m}$ or greater and a thickness of either $12.5 \mu \mathrm{m}$ or $6.25 \mu \mathrm{m}$. Since a $12.5 \mu \mathrm{m}$ flexure $125 \mu \mathrm{m}$ long cannot be used in the $3^{\text {rd }}$ and $4^{\text {th }}$ joints of the fourbar because the motions are too large, this puts yet another constraint on the allowable dimensions of the flexures. Taking all these factors into account, we finally arrive at the following flexure dimensions:

\begin{tabular}{|c|c|c|c|c|}
\hline & 1 & 2 & 3 & 4 \\
\hline $\mathrm{l}(\mu \mathrm{m})$ & 125 & 125 & 125 & 125 \\
\hline $\mathrm{w}(\mathrm{mm})$ & 3 & 3 & 4 & 4 \\
\hline $\mathrm{t}(\mu \mathrm{m})$ & 6.25 & 6.25 & 12.5 & 12.5 \\
\hline
\end{tabular}

\section{CALCULATION OF PARALLEL STIFFNESS}

The parallel stiffness of a flexure mechanism is easy to calculate if we assume that each flexure is an ideal rotational joint with a constant rotational stiffness. For small rotational angles, the rotational stiffness of a flexure is given as

$$
k_{\text {flex }}=\frac{E I}{l}
$$

In an $N$-link mechanism, the total $P E$ stored in the flexures of the mechanism is given by:

$$
P E_{\text {tot }}=\sum_{i=1}^{N} 1 / 2 k_{i} \gamma_{i}^{2}
$$

where $k_{i}$ is the stiffness of the $i^{t h}$ flexure calculated using (4) and $\gamma_{i}$ is the total deflection of the $i^{\text {th }}$ flexure. To find the rotational stiffness of the mechanism with respect to the driving actuation angle, call it $\alpha$, we need to first find the restoring force for a given value of $\alpha$. This is given as the gradient of the $P E$ as

$$
\begin{aligned}
F & =\frac{\partial P E}{\partial \alpha} \\
& =\sum_{i=1}^{N} k_{i} \gamma_{i} \frac{\partial \gamma_{i}}{\partial \alpha}
\end{aligned}
$$

For small displacements about some nominal value of $\alpha=\alpha_{0}$, the stiffness of the mechanism is given as:

$$
\begin{aligned}
k_{e q} & =\left.\frac{\partial F}{\partial \alpha}\right|_{\alpha=\alpha_{0}} \\
& =\sum_{i=1}^{N} k_{i}\left(\frac{\partial \gamma_{i}}{\partial \alpha}\right)^{2}+k_{i} \gamma_{i} \frac{\partial^{2} \gamma_{i}}{\partial \alpha^{2}}
\end{aligned}
$$

Note that it doesn't make sense to use a stiffness number for large motions. For the complete non-linear dynamics, we should directly use the value of the restoring force $F$ according to (6).

\section{A. Effect of flexure pre-stress}

Note that in (7), $\gamma_{i}$ represents the total displacement of the flexural from its strain-free state. This displacement depends not just on the kinematics of the mechanism but also on the fabrication method, which might impart a pre-stress to some 
of the flexures without any driving actuation. In other words, $\gamma_{i}$ in (7) can be thought of as:

$$
\gamma_{i}=\gamma_{i}^{\mathrm{kin}}+\gamma_{i}^{\mathrm{fab}}
$$

Here, $\gamma_{i}^{\text {kin }}=0$ when the driving angle $\alpha=0 . \gamma_{i}^{\text {fab }}$ is the pre-stress in the joint which results from fabrication. Note that it is constant for a given fabricated part. Since

$$
\frac{\partial \gamma_{i}}{\partial \alpha}=\frac{\gamma_{i}^{\text {kin }}}{\partial \alpha},
$$

therefore, the first term in (7) depends purely on the kinematics and not on the initial pre-stress due to fabrication. Thus, we need to take the initial pre-stress of the flexure into consideration only for those flexures for which we have

$$
\frac{\partial^{2} \gamma_{i}}{\partial \alpha^{2}} \neq 0
$$

about the nominal operating point.

We can see the effect of pre-stressing on the differential stiffness in Fig. 9. As we can see there is almost a $100 \%$ increase in the differential stiffness when the $\theta_{y}$ flexure is pre-stressed by $90^{\circ}$. To minimize the effect of flexure prestress, we have found that its usually a good idea to "cure" the whole mechanism at a heightened temperature after the fabrication is complete.

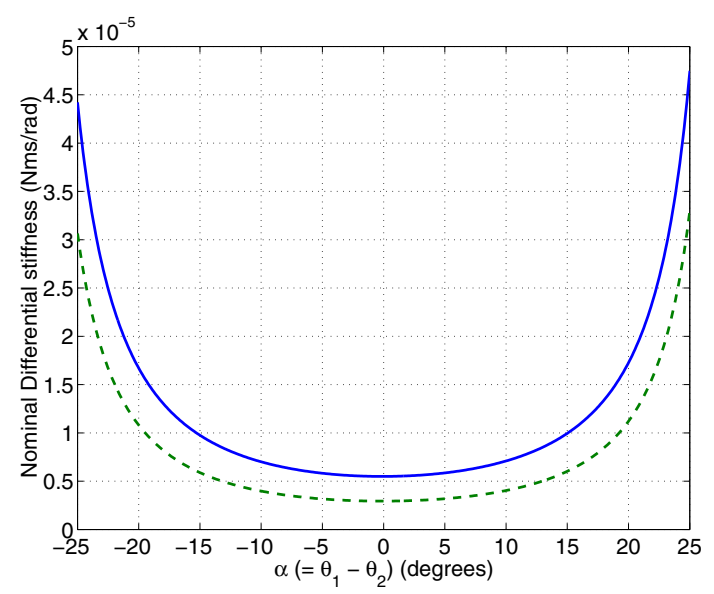

Fig. 9. Effect of flexure pre-stress. The solid line represents the stiffness of the differential w.r.t $\alpha$ when $\theta_{y}^{0}=0^{\circ}$ and the dotted line represents the stiffness with $\theta_{y}^{0}=90^{\circ}$.

\section{B. Effect of misalignment}

Detailed geometric analysis turns out to be extremely important in mechanisms where certain geometric constraints need to be met for the mechanism to move.

Consider the wing differential mechanism of the MFI shown in Fig. 2. It consists of two links labeled 1 and 4 which are actuated independently via two fourbars. They are connected together by a series of 3 links which are interconnected by simple flexural elements. The basic idea is that when the links 1 and 4 are moved in phase, then the middle plate 2 rotates along with them about their common axis $Z$. However, when 1 and 4 move out of phase, the link 4 in addition to rotating about $Z$ also rotates about the other axes $X, Y$ and $W$.

The kinematics of the differential in the absence of any misalignments has already been derived in previous work [12] as

$$
\begin{aligned}
& \theta_{x}=\sin ^{-1}(\lambda \sin \alpha) \\
& \theta_{y}=\beta-\cos ^{-1}(\lambda \sin \beta)
\end{aligned}
$$

where $\beta=\tan ^{-1}\left(\frac{1}{\lambda \cos \alpha}\right)$

To emphasize the importance of alignment in the differential mechanism, it is appropriate to step back and start from one of the most simplistic concerns of kinematics, namely to find the available degrees of freedom in the mechanism. If we simply "count" the number of degrees of freedom in the mechanism using Gruebler's criterion:

$$
\begin{aligned}
N_{\text {diff }} & =6 * N_{\text {links }}-5 * N_{\text {flexures }} \\
& =6 * 4-5 * 5 \\
N_{\text {diff }} & =-1
\end{aligned}
$$

What this means is that an arbitrary closed 4 linked (5 with ground link included) spatial mechanism with flexures along arbitrary axes will be "jammed". In the MFI differential, the two extra degrees of freedom come from two of the flexural constraints becoming redundant in the presence of geometric constraints.

Consider the purely kinematic diagram of the differential shown in Fig. 2. From a kinematic perspective, the mechanism would jam completely if the flexural axes represented by the axes $\theta_{1}, \theta_{2}, \theta_{x}, \theta_{y}$ and $\theta_{w}$ were to not intersect exactly at the single point shown in the figure as the origin. In reality, the flexural compliance allows some movement albeit at the expense of added unwanted stiffness.

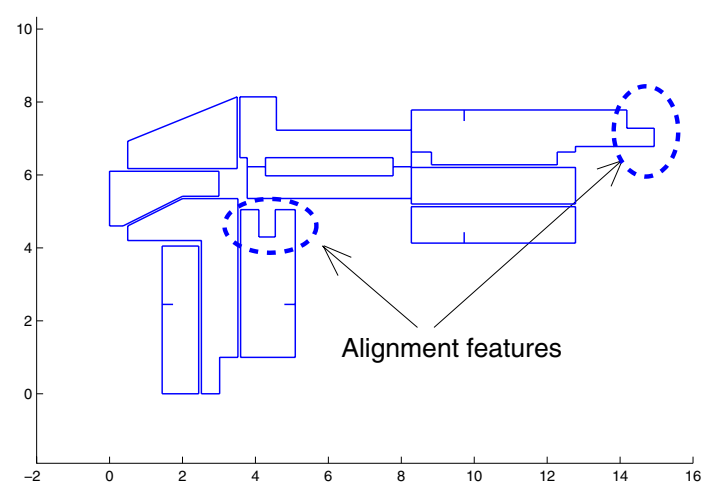

Fig. 10. Cut pattern of differential on Carbon fiber

To ensure the kind of alignments we need, we utilized a fabrication design in which the complete differential is cut out 
under the laser machine as a single part. Fig. 10 shows the final design of the differential cuts. The alignment is critical enough that we needed to account for the width of the laser beam which cuts out the pattern on the carbon fiber sheet. In addition to this, we also fabricated the output links of the fourbar links as part of the differential mechanism to ensure the alignment mentioned above. Features are added to the differential design which mate and thus ensure good alignment. The latest differential mechanism exhibits a parallel stiffness which is just 1.5-1.6 times the predicted stiffness with perfect alignment. This is quite a gain from previous designs which were almost 40-50 times the predicted stiffness.

\section{Conclusions}

To summarize, we need to account for the following factors while designing a flexural mechanism using the new carbon fiber fabrication techniques:

- Analyze the forces transmitted through the flexures in the fourbar and design the flexures to always point in the direction of the transmitted forces.

- For flexural mechanisms, it is also very important to design the flexures to never buckle under typical operating conditions.

- It is important to account for flexure pre-stressing in the calculation of mechanism stiffnesses.

- For 3D mechanisms where mobility arises from geometric constraints, it is important to analyze for the effect of misalignment and design the structure in a manner which ensures proper alignment.

Fig. 11 shows a photo of the latest MFI thorax mechanism which utilizes these various design rules in construction. For clarity, we also show the a $3 \mathrm{D}$ sketch of the mechanism in Fig. 12.

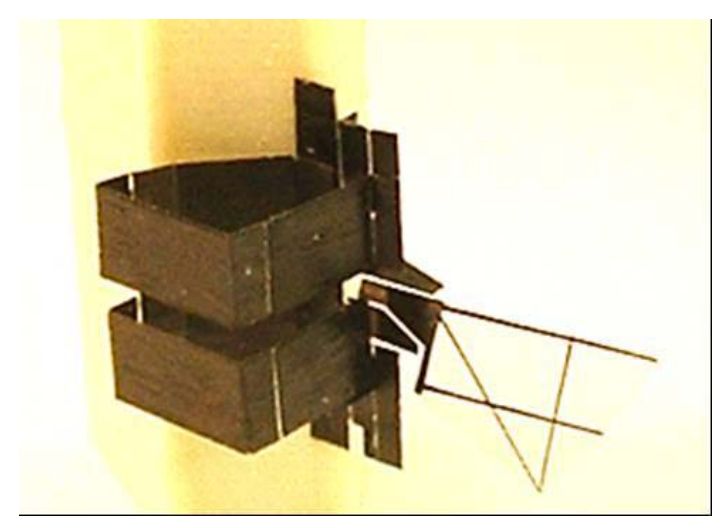

Fig. 11. Photo of the latest MFI differential

\section{ACKNOWLEDGMENTS}

The authors would like to thank Erik Steltz, Ranjana Sahai and Robert Wood for insightful discussions and invaluable help.

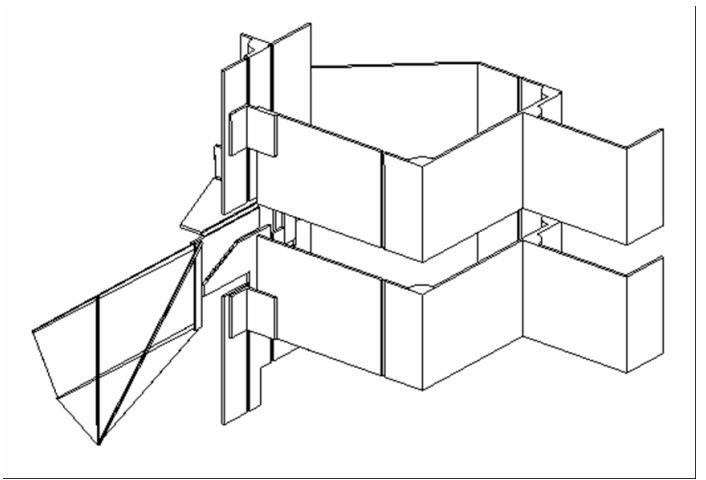

Fig. 12. 3D sketch of the latest MFI differential

\section{REFERENCES}

[1] S. Kota. Design of compliant mechanisms, application to MEMS. In SPIE Conference on Smart Electronics and MEMS, Newport Beach, CA, Mar 1999.

[2] B. Yi, G. B. Chung, H. Y.Na, and W. K. Kim. Design and experiment of a 3-DOF parallel micromechanism utilizing flexure hinges. IEEE Transactions on Robotics and Automation, 19(4), Aug 2003.

[3] M. Carricato, V. Parenti-Castelli, and J. Duffy. Inverse static analysis of a planar system with flexural pivots. Journal of Mechanical Design, 123:43-50, March 2001.

[4] B. H. Kang, J. T. Wen, N. G. Dagalakis, and J. J. Gorman. Analysis and design of parallel mechanisms with flexure joints. In Proc. IEEE Int. Conf. on Robotics and Automation, April 2004.

[5] L. L. Howell and A. Midha. A method for the design of compliant mechanisms with small-length flexural pivots. Journal of Mechanical Design, 116:280-90, March 1994.

[6] M. Goldfarb and J. E. Speich. A well-behaved revolute flexure joint for compliant mechanism design. Journal of Mechanical Design, 121(3):424-429, September 1999.

[7] R. S. Fearing, K. H. Chiang, M. H Dickinson, D. L. Pick, M. Sitti, and J. Yan. Wing transmission for a micromechanical flying insect. In Proc. IEEE Int. Conf. on Robotics and Automation, pages 1509-1516, San Francisco, CA, April 2000.

[8] R. J. Wood, S. Avadhanula, and R. S. Fearing. Microrobotics using composite materials. In Proc. IEEE Int. Conf. on Robotics and Automation, Taipei, Taiwan, May 2003.

[9] E. Shimada, J. A. Thompson, J. Yan, R. J. Wood, and R. S. Fearing. Prototyping millirobots using dextrous microassembly and folding. In Symp. on Microrobots ASME Int. Mech. Eng. Cong and Expo., Orlando, FL, November 2000.

[10] R. Sahai, J. Lee, and R. S. Fearing. Semi-automated micro assembly for rapid prototyping of a one DOF surgical wrist. In IEE Conf. on Intelligent Robots and Systems, Las Vegas, NV, Oct 2003.

[11] R. Sahai, E. Steltz, and R. S. Fearing. Carbon fiber components with integrated wiring for millirobot prototyping. In (submitted to) Proc. IEEE Int. Conf. on Robotics and Automation, Barcelona, Spain, April 2005.

[12] S. Avadhanula, R. J. Wood, D. Campolo, and R. S. Fearing. Dynamically tuned design of the MFI thorax. In Proc. IEEE Int. Conf. on Robotics and Automation, pages 52-9, Washington, DC, May 2002.

[13] S. Avadhanula, R. J. Wood, E. Steltz, J. Yan, and R. S. Fearing. Lift force improvements for the micromechanical flying insect. In IEE Conf. on Intelligent Robots and Systems, Las Vegas, NV, Oct 2003.

[14] X. Deng and S. Avadhanula. Biomimetic underwater microbotic boxfish with oscillating fin propulsion: System design and force measurement. In (submitted to) Proc. IEEE Int. Conf. on Robotics and Automation, Barcelona, Spain, 2005. 